\title{
- Nanostructures in GaAs Fabricated by Molecular Beam Epitaxy
}

\author{
Loren N. Pfeiffer, Kenneth W. West, Robert L. Willett, \\ Hidefumi Akiyama, and Leonid P. Rokhinson
}

We review three novel techniques whereby the highly uniform twodimensional films produced by molecular beam epitaxy (MBE) can be further patterned into nanowires or nanostructures having quantum confinement in all three dimensions. These techniques have the potential of greatly extending the power and utility of the MBE method.

(C) 2005 Lucent Technologies Inc.

\section{Introduction}

Molecular beam epitaxy (MBE) is essentially a controlled evaporation from various elemental sources in high vacuum onto a temperature-controlled single crystal substrate. The idea of epitaxy is that in the growing layer the newly arriving atoms incorporate at the precise positions established by the immediately preceding atomic layer. This epitaxial relationship extends even to a hetero-interface where a semiconducting GaAs layer abruptly changes to the higher bandgap more insulating material, AlAs, or to the alloy AlGaAs. Because GaAs, AlAs, and AlGaAs have sufficiently similar crystal structures to one another, MBE can produce single crystal layers of uniform and precisely controlled nano-thickness with no mismatched chemical bonds at the hetero-interfaces.

In some sense these nano-thick epitaxial films are indeed ideal two-dimensional (2D) nanostructures; however, in the remainder of this paper, we will use the word nanostructure to mean structures that have dimensions smaller than $100 \mathrm{~nm}$ in at least two rightangle directions. Thus, all MBE films will require further processing of some kind if they are to be fabricated into nanostructures as the word is intended here. In this paper, we highlight, three of the various methods for fabricating nanostructures from GaAs-AlGaAs MBE films that we have been recently exploring at Bell Labs.

\section{Fabrication of Nanostructures in GaAs Defined by Scanned Local Oxidation on the Surface of a Very Shallow 2D Electron or Hole System}

In these nanostructures, the $2 \mathrm{D}$ carriers are depleted only under the locally oxidized areas of the scanned tip [3]. This divides the system into several conducting 2D regions separated by insulating walls. The particular nanostructure we fabricated using this technique is the first nanodevice that is able to sort ballistic holes into their separate spin-up and spindown components [6]. This capability of sorting carriers by their spin alignment in a magnetic field is prerequisite to making the proposed new field of spin-electronics or spintronics a reality. We use the fact that, in a AlGaAs-GaAs semiconductor heterostrucure, the spin-up and spin-down holes at the Fermi energy have, in a magnetic field, slightly differing cyclotron orbits because of their differing spinorbit interactions. This spin-separation experiment requires a host of novel nanostructure techniques

Bell Labs Technical Journal 10(3), 151-159 (2005) @ 2005 Lucent Technologies Inc. Published by Wiley Periodicals, Inc. Published online in Wiley InterScience (www.interscience.wiley.com). • DOI: 10.1002/bltj.20110 
because the spin-up to spin-down orbit separations typically translate to only about $100 \mathrm{~nm}$ of lateral displacement.

As a first step in the nanofabrication, we designed a conceptually novel MBE quantum well structure for $2 \mathrm{D}$ holes that is modulation-doped from below and surface-compensated from above [7]. This allows us to reduce the quantum-well-to-sample-surface distance to only $350 \mathrm{~A}$, while maintaining an undoped modulation-doping setback in excess of $500 \mathrm{~A}$ from below. The valance band energy diagram versus depth into the AlGaAs-GaAs sample is shown in Figure 1.

The symbol $E_{\mathrm{f}}$ is the Fermi level, and the vertical axis is depth within the sample referenced to the $150 \AA$ wide GaAs quantum well located $350 \AA$ A below the sample surface. These shallow 2D quantum well structures have achieved 2D hole mobilities of $600,000 \mathrm{~cm}^{2} /$ Vsec at various predetermined hole densities, which typically are $1.4 \times 10^{11} \mathrm{~cm}^{-2}$.

The interesting thing about this structure is the extreme sensitivity of the 2D hole density to the thickness of the $150 \AA$ GaAs layer between the upper $\mathrm{Si}$ $\delta$-doping and the sample surface. The left side of Figure 1 shows the band diagram of the structure after removing $70 \AA$ of the surface GaAs layer by GaAs conversion to gallium oxide. This small thickness change in the GaAs cap layer completely shuts off all valance
Panel 1. Abbreviations, Acronyms, and Terms

1D-One-dimensional

2D-Two-dimensional

AFM-Atomic force microscope

HG-Hole gas

MBE-Molecular beam epitaxy

PMMA—Polymethyl methacrylate

SEM-Scanning electron micrograph

TEM-Transmission electron micrograph

band conduction in the sample by lifting the bottom of the quantum well above the Fermi level. We make use of this extreme sensitivity by installing the sample in an atomic force microscope (AFM), where a microtip under voltage bias is scanned in a humid atmosphere just above the sample surface. Wherever the tip is scanned, the surface becomes locally oxidized in a strip $300 \AA$ wide and about $100 \AA$ deep. This effectively moves the surface states $100 \AA$ closer to the quantum well in the strip-oxidized region, which is enough to locally lift the quantum well above the Fermi level of the 2D hole gas (HG) and cause the well to fully deplete everywhere the oxidation probe was scanned.

The surface oxidation walls below which the 2D HG is depleted are shown in the $5 \mu \mathrm{m} \times 5 \mu \mathrm{m}$ inset of Figure 2, where region 1 is the injector, the white (top)
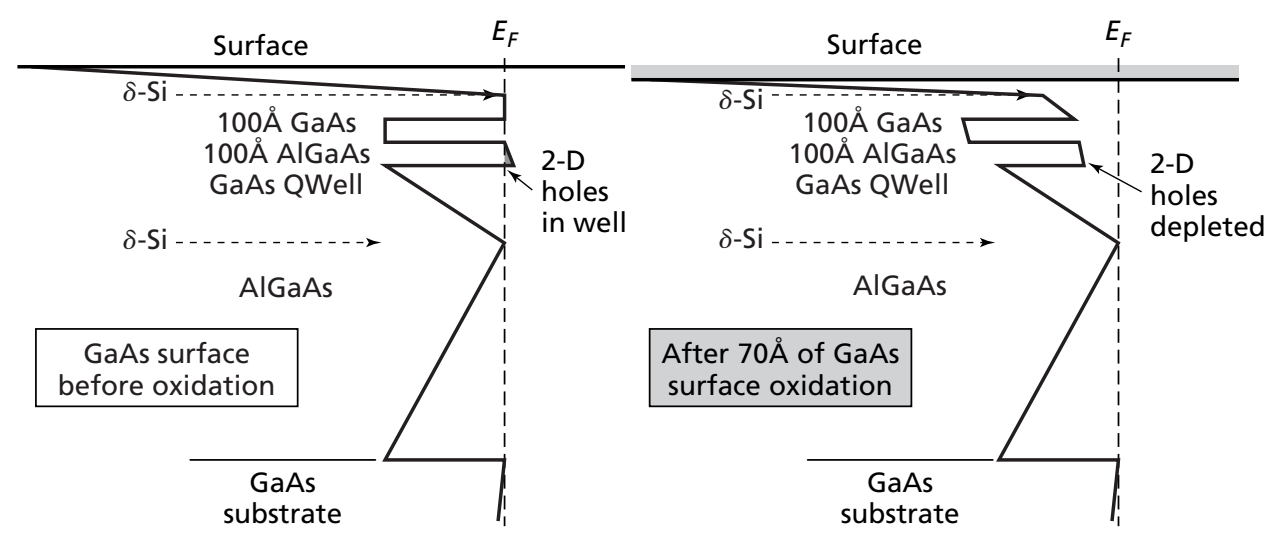

By bringing the sample $7 \mathrm{~nm}$ closer, the oxidation depletes the shallow quantum well of holes.

Figure 1.

Poisson-Schrodinger calculations of the valence band energy diagram of very shallow quantum well sample before and after $7 \mathrm{~nm}$ of surface oxidation. 


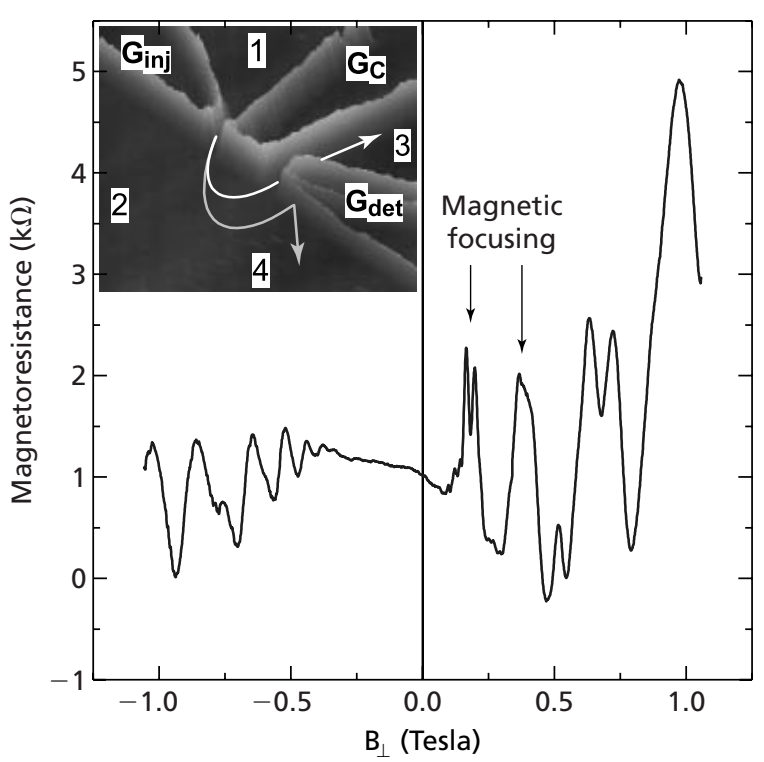

AFM-Atomic force microscope

Inset: AFM nanograph of the sample. The lighter walls are local oxidation barriers formed using a scanned tip under bias in a humid atmosphere. The white (top) and gray (bottom) curves represent differing cyclotron orbits of the spin-up and spin-down ballistic holes in a perpendicular magnetic field.

Figure 2.

The $36 \mathrm{mT}$ spin-up and spin-down orbit separation of ballistic holes at $0.18 \mathrm{~T}$.

and gray (bottom) curves are the trajectories of the two spins in a perpendicular magnetic field, and V3-V4 is the detector voltage plotted in the main figure. The magnetic focusing doublet at $\mathrm{B}_{\perp}=0.18 \mathrm{~T}$ (Figure 3) shows a separation of $36 \mathrm{mT}$. This corresponds to a measured spin up-to-down orbit separation of only $120 \mathrm{~nm}$. A separate experiment using an additional 3.3 T in-plane B-field caused the higher peak in the doublet to disappear, demonstrating preferential selection for spin-down 2D holes [6].

The scanned-oxide fabrication techniques employed here are very general. Because they allow any shallow 2D conducting quantum layer to be divided into various nanoscale conducting regions separated by highly insulating quantum barriers, they raise exciting possibilities for applying quantum effects to useful devices. For example, to make a quantum-scale wire with macroscopic contact pads, one would

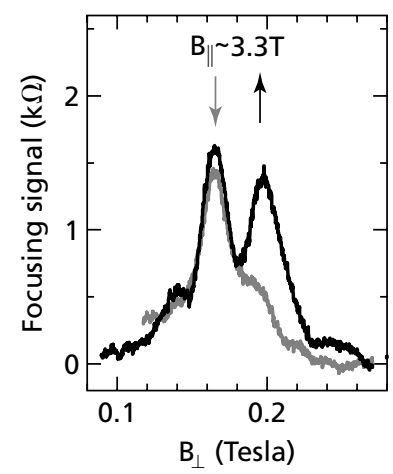

The gray trace is attributed to the trajectories of spin-down holes.

Figure 3.

Demonstration of spin selection of 2D holes at the $0.18 T$ magnetic focusing peak using an additional in-plane magnetic field.

simply have the oxidizing tip draw two insulating oxide barriers in an hourglass shape with an extended waist area. The large portions of the hourglass become the electrical contact pads, and the extended waist becomes the quantum wire under study. The final point to note is that these scanned oxide techniques can readily be combined with conventional integrated circuit patterning, so that the large-scale areas are defined by the usual lithographic and chemical techniques, leaving only the more specialized quantum and nanoscale regions to be defined by the oxide-scanned probe.

\section{Fabrication of Nanoscale Metal Wires Defined by Nanomolds Fabricated from GaAs-AIGaAs MBE Layers}

This technique exploits the special strength of molecular beam epitaxy for fabricating GaAs and AlGaAs layers whose thicknesses are uniform on the micro-scale. The initial process steps for the fabrication are summarized in Figure 4. First the MBE process is used to grow alternating layers of nanoscale-thick GaAs and AlGaAs, and then the GaAs substrate is removed from the MBE machine and cleaved in the open atmosphere exposing the [110] cleavage surfaces that contain a cross-section of the various layers. 

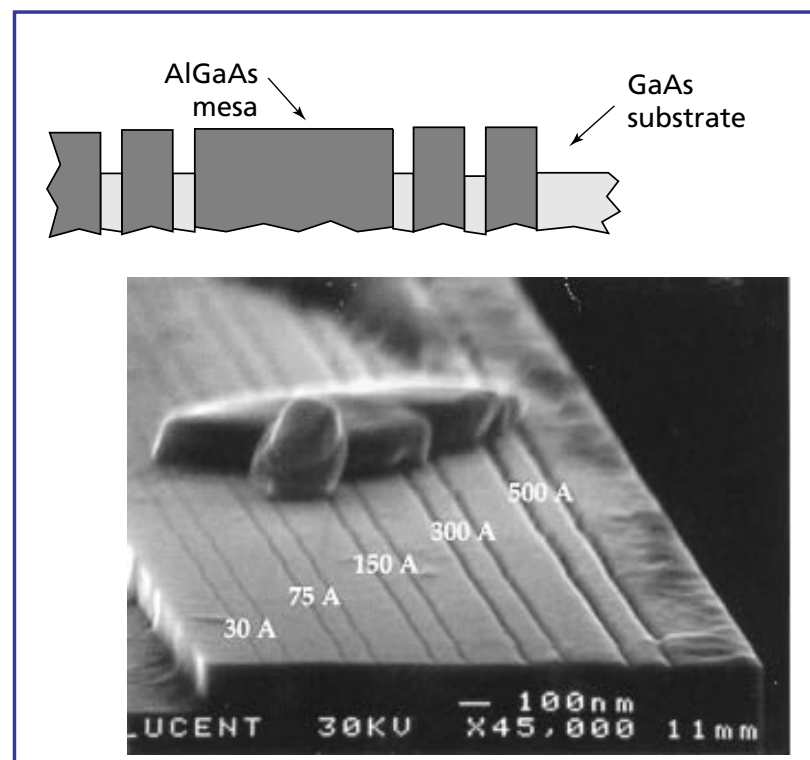

Figure 4.

Scanning electron micrograph of the air-cleaved edge of an AlGaAs-GaAs multilayer after a selective chemical etch that removes only the GaAs wells to a $50 \mathrm{~nm}$ depth.

Then, using a chemical etch that preferentially attacks GaAs compared to AlGaAs, the GaAs layers and substrate are etched-back to obtain the grooved structure shown in the upper part of the figure. The lower part of the figure is a scanning electron micrograph (SEM) showing the cleaved edge of an actual GaAs-AlGaAs sample with five pairs of nanogrooves ranging in width from $3 \mathrm{~nm}$ to $50 \mathrm{~nm}$.

The next process step is an optional deposition and patterning of polymethyl methacrylate (PMMA) photoresist shown in light gray in Figure 5, followed by the deposition of the evaporated metal, shown in white, that will form the nanowires. The final step is an ion beam etch directed at a low angle with respect to the [110] cleave-surface. This does not affect the metal wire protected by the deep AlGaAs-walled trench, but it does remove the excess metal deposited on top of the AlGaAs separation layers, thus assuring that the metal nanowires are electrically isolated from the rest of the structure. Electrical contact to the nanowires is formed by means of metal contact strips lying normal to the wires and defined using lithography and metal liftoff. This procedure is conventional

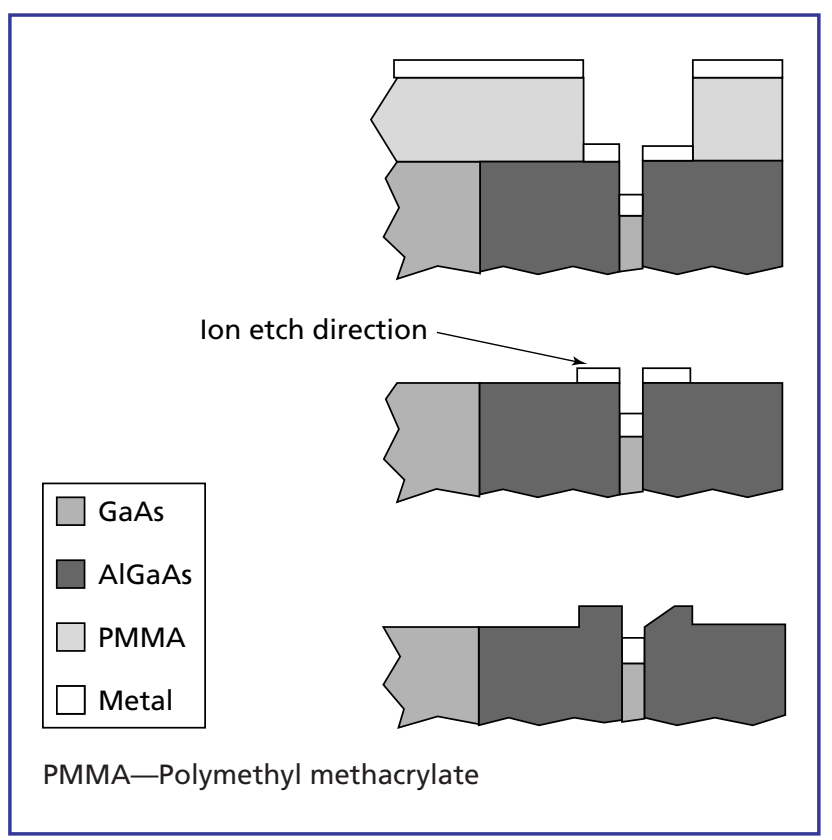

Figure 5.

Process diagram showing metal deposition and ionetch removal steps needed in forming metal nanowires in the GaAs grooves on the air-cleaved sample edge.

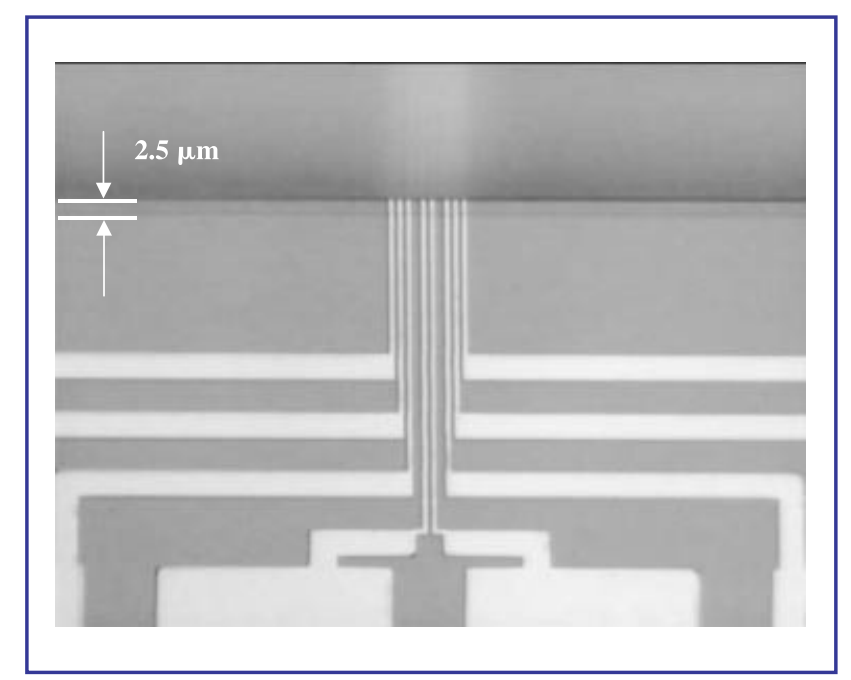

Figure 6.

Micrograph of the metal contacting leads to the metal nanowires.

except for the fact that is must be done on the $500 \mu \mathrm{m}$ wide cleaved edge. Figure 6 shows the contacts; the unseen nanowire or wires run horizontally between the contact strips within the wider AlGaAs separation barriers shown as a dark contrast stripe $2.5 \mu \mathrm{m}$ from the edge of the sample. 


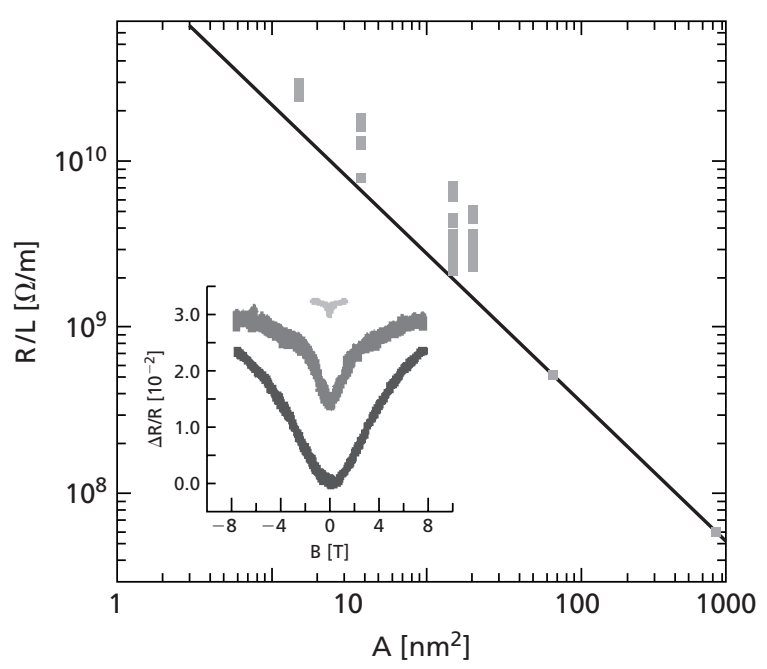

The inset demonstrates quantum corrections observed at $\mathrm{T}=4.2 \mathrm{~K}$ in nanowires of width $5 \mathrm{~nm}$ (black trace), $20 \mathrm{~nm}$ (dark gray trace), and $88 \mathrm{~nm}$ (light gray trace).

Figure 7.

Data plot of resistance per unit length of nanowires versus their cross-sectional area.

Using these techniques, we have fabricated continuous metal nanowires of AuPd that are several microns in length and as narrow as $3 \mathrm{~nm}$ in crosssection [4]. These wires are remarkably robust. They have survived repeated cycling to cryogenic temperatures and DC current densities of up to $10^{9} \mathrm{~A} / \mathrm{cm}^{2}$ before becoming discontinuous.

The data points on Figure 7 plot room temperature for terminal-resistance measurements of various AuPd nanowires made by this method versus the measured cross-sectional area of the wire. The solid line is the resistance expected for the various wires based on a calculation of the wire area and the measured 2D resistivities of AuPd films code-posited with the wires. The generally good agreement between the two establishes that we are repeatedly able to make metal wires with uniform cross sections as small as $15 \mathrm{~nm}^{2}$, which corresponds to the designed wire width of $3 \mathrm{~nm}$.

The inset of Figure 7 demonstrates that at the smallest wire diameters achieved with this technique, we already see significant quantum corrections to the wire conductivity at $4.2 \mathrm{~K}$. Shown here is magneto- resistance data for AuPd metal wires $88 \mathrm{~nm}$ (top), $20 \mathrm{~nm}$ (center), and $5 \mathrm{~nm}$ (lower) in diameter. In quantum sized metal wires at low temperatures, pair-loops of time-reversed electron scattering trajectories can contribute coherently to the wire conductance [5]. Magnetic flux suppresses the contribution of such loops. Thus, at zero magnetic field the quantum coherent scattering electron trajectories contribute; however, as the magnet field is raised, the quantum coherence is suppressed and the wire resistance rises for the smallest wire by several percent. As the wire diameters become even smaller and the temperature is lowered further, such quantum coherence effects are expected to grow substantially.

\section{Fabrication of a Nanostructure Laser from a Single Quantum Wire}

This nanostructure is fabricated entirely by MBE in a three-step process that we have named "cleavededge overgrowth" [1]. As shown in Figure 8, the first step is a conventional MBE growth of a GaAs quantum well in the (100) crystal plane sandwiched between two AlGaAs barriers. The substrate wafer is then removed from the MBE machine and thinned from the backside to about $90 \mu \mathrm{m}$ total thickness. It is then cleaved in air into square pieces $10 \mathrm{~mm}$ on side, with additional tick marks at the site of a future cleave to be done in the MBE. These pieces are mounted using Ga solder against a low tantalum wall in the MBE machine so that the top edge of each piece faces the source furnaces. When the MBE is again brought to the proper growth conditions, a mechanical arm (step 2) sweeps across the freely standing pieces, cleaving them at the pre-established tick marks and thereby exposing a fresh (110) crystalline surface. Step 3 is started within a few seconds as the MBE source shutters are opened and the initiation of the second MBE growth begins on the newly exposed (110) cleaved edge.

If the second MBE growth is also a quantum well followed by an AlGaAs barrier, then we will have fabricated a nanostructure with two quantum wells that intersect at right angles. The intersection of two such wells has been shown to be a one-dimensional (1D) wire-like trap for electrons, for holes, and for 


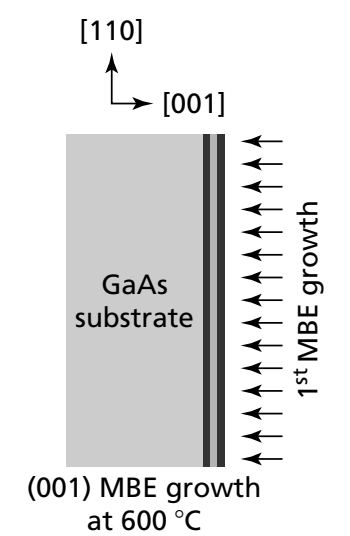

Step 1

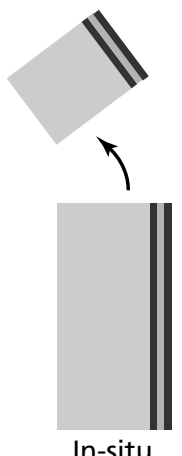

Cleave

Step 2

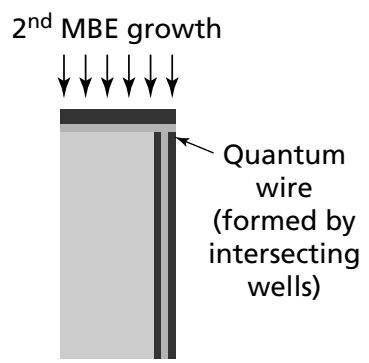

(110) MBE growth at $500^{\circ} \mathrm{C}$

Step 3

MBE-Molecular beam epitaxy

Figure 8.

Schematic diagram of the cleaved-edge overgrowth molecular beam epitaxy process used in this case to fabricate intersecting quantum wells.

excitons [8]. Figure 9 illustrates the probability contours for finding a bound electron or bound hole at such an intersection. The binding of any of these particles to the line-like intersecton is completely quantum mechanical in nature. Its origin is that only at the T-intersection can the quantum mechanical wavefunction lower its energy by speading out into three possible lobes.

Having established how we can form a quantum wire by cleaved-edge-overgrowth MBE, it now only remains to show how we embedded our wire into an appropriate optical cavity that comprises the first laser that operates from the quantum wire ground state. This is shown in Figure 10. The laser consists of a T-shaped optical cavity with a $1000 \mathrm{~nm}$ wide optical stem of AlGaAs at $35 \% \mathrm{Al}$ fraction, intersecting an optical cross-arm $111 \mathrm{~nm}$ wide of AlGaAs with Al fraction $10 \%$. As may be seen in the figure this optical $\mathrm{T}$-structure is itself embedded in an AlGaAs alloy of $\mathrm{Al}$ fraction $50 \%$. The optical wire-like confinement modes are shown as gray contours in the figure. The excitonic quantum wire consists of a $14 \mathrm{~nm}$ wide AlGaAs stem well with a $7 \% \mathrm{Al}$ alloy fraction, intersecting a crossarm of GaAs $6 \mathrm{~nm}$ wide. The confinement contours for

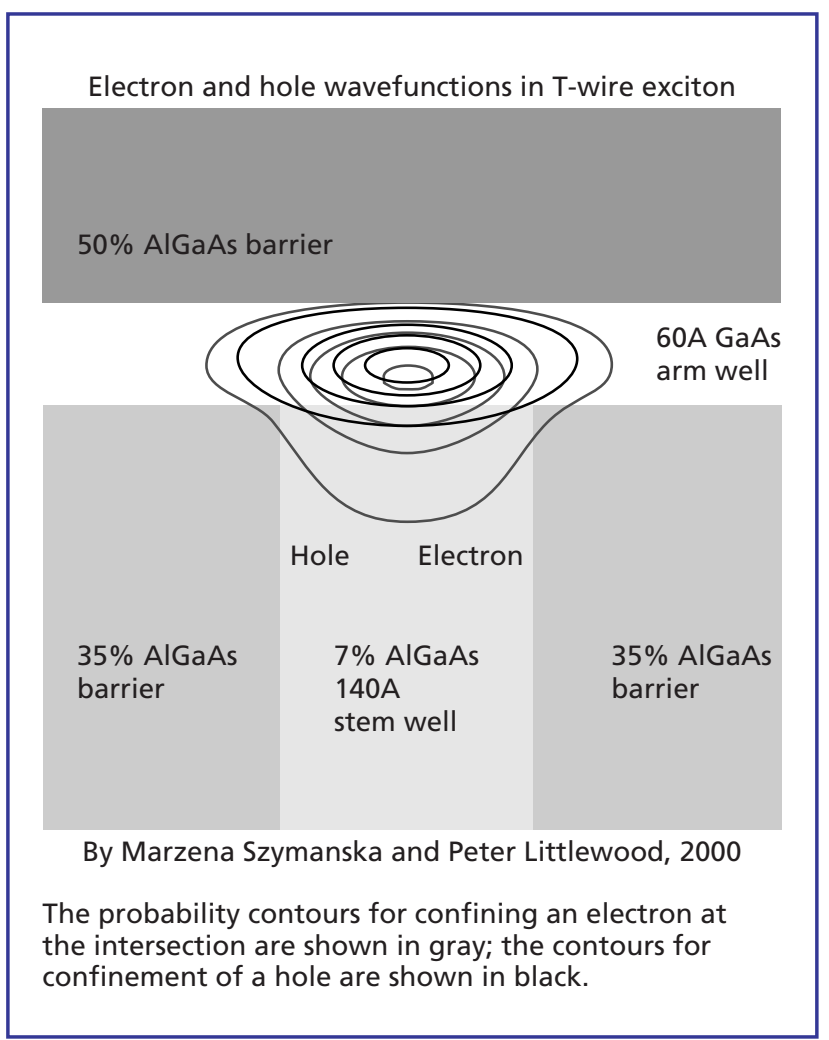

Figure 9.

Formation of a quantum wire at the right-angle intersection of two quantum wells. 


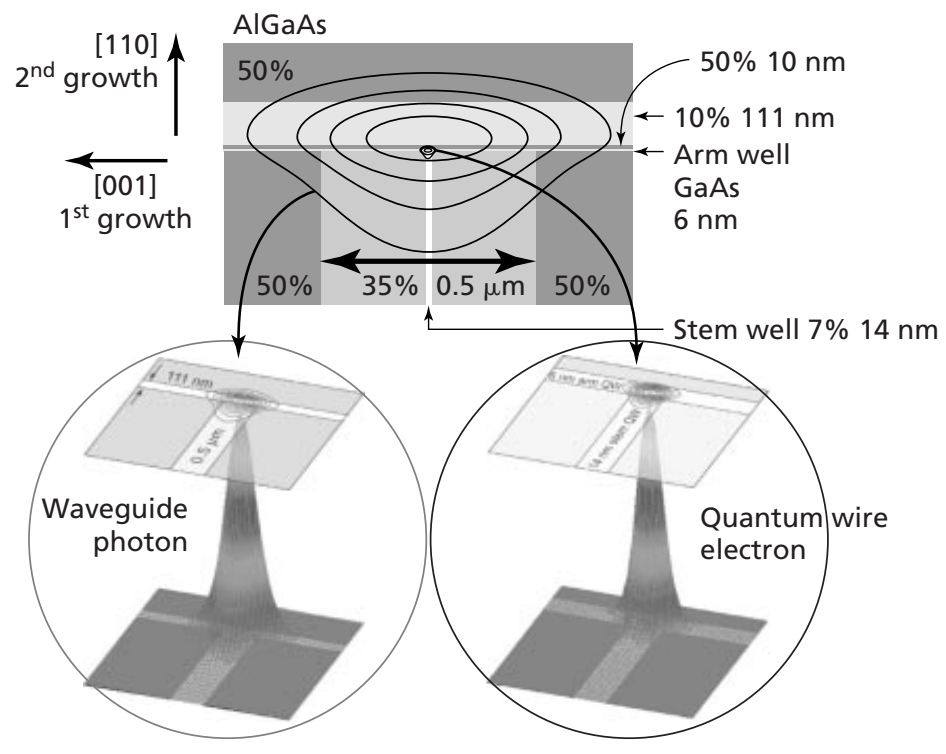

The exciton confinement probability contours are shown in black; the contours for confinement of a photon are shown in gray.

\section{Figure 10.}

Schematic diagram of the structure of a single wire laser.

excitons at the quantum-wire intersection are shown in black.

Figure 11 is a transmission electron micrograph (TEM) of a very similar T-laser structure fabricated in our laboratory with multiple stem wells and multiple excitonic wires. The T-intersections and the cross-arm for optical confinement are clearly visible.

An example of the photoluminescence (PL) uniformity of our single quantum wire along its length is shown in Figure 12. These are edge-emitting lasers typically $0.5 \mathrm{~mm}$ long expected to laser-emit along the quantum wire axis, with Au mirrors on the aircleaved end-facets. This PL data was taken by scanning a $0.5 \mu \mathrm{m}$ dia-micro-focused excitation spot in $0.5 \mu \mathrm{m}$ steps along the entire length of the quantum wire. The wire PL at $1.581 \mathrm{eV}$ is very sharp at $0.9 \mathrm{meV}$ linewidth, and very uniform along the entire wire except for a very few localized excitonic states presumably caused by local fluctuations in the quantum wire width. The wider PL emission at $1.635 \mathrm{eV}$ is due to excitation of the stem well.

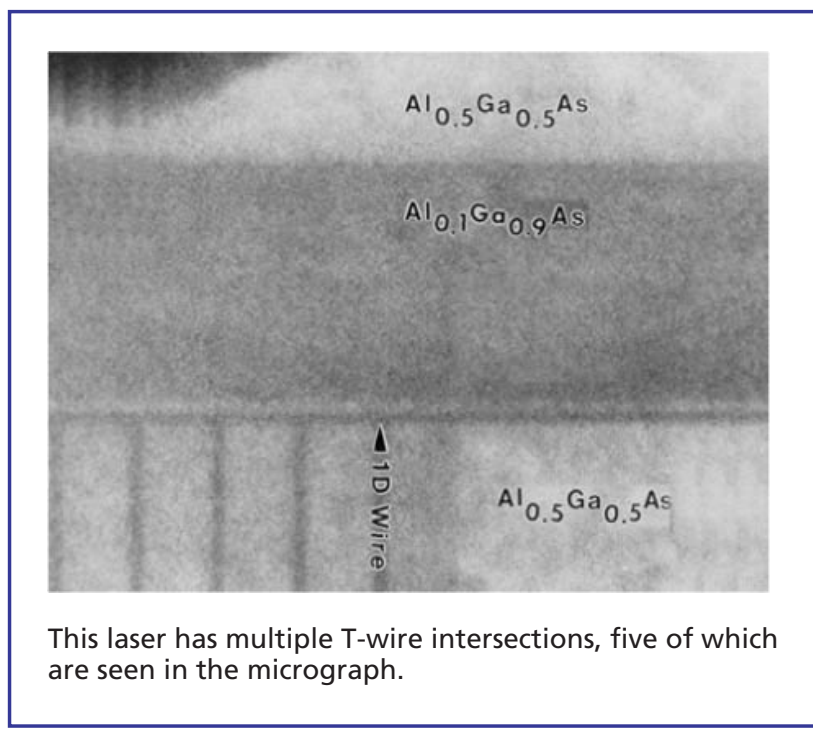

Figure 11.

Transmission electron micrograph showing part of the cross section of an actual quantum wire T-laser.

Figure 13 shows the laser emission characteristics of our single-quantum-wire edge-emitting laser [2]. These characteristics were obtained by optical pumping along 


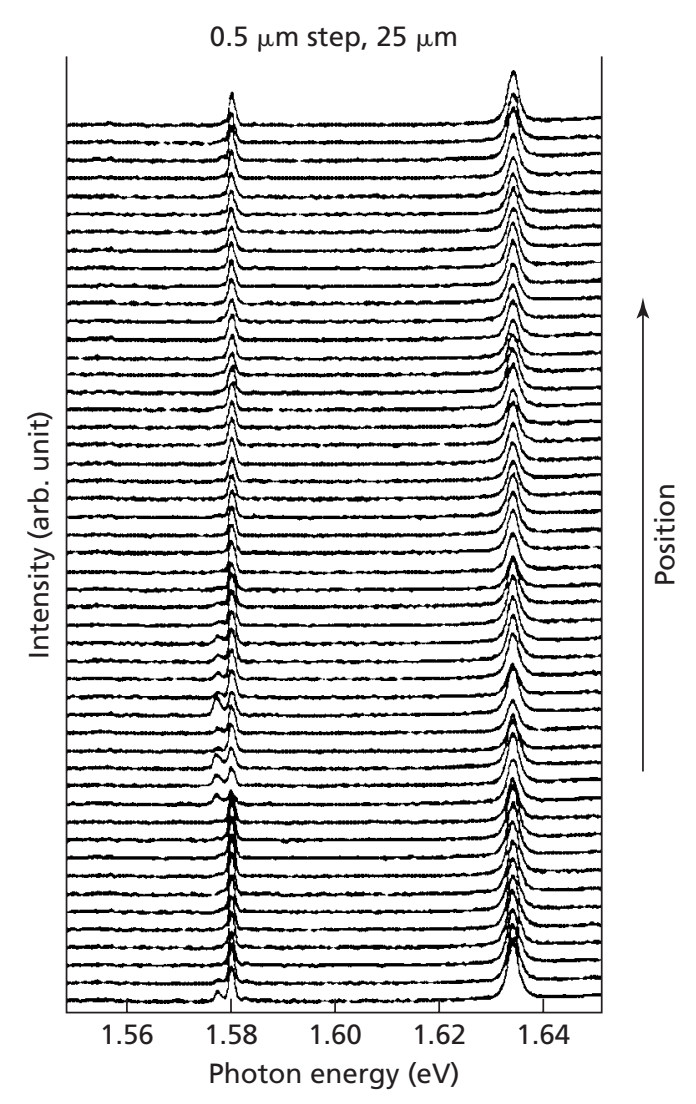

Other scans using $5 \mu \mathrm{m}$ intervals demonstrate that the entire $0.5 \mathrm{~mm}$ laser nanowire is continuous with a PL line width of 0.9 meV FWHM.

Figure 12.

A photoluminescence scan using a $1 \mu \mathrm{m}$ excitation spot moving at $0.5 \mu \mathrm{m}$ intervals for $\mathbf{2 5} \mu \mathrm{m}$ along a single $T$-wire.

the length of the wire at $4.2 \mathrm{~K}$ for a $5 \mathrm{~mm}$ long single quantum wire laser with air-cleaved facets that were Au-coated to form mirrors. Notice that the optical pumping threshold power to initiate lasing is only $5 \mathrm{~mW}$. Notice further that we are able to raise the optical pumping power by more than a factor of 50 above this value and that, even at this extreme pumping level, we obtain an energy stable laser output with a tiny shift of 1.8 meV compared to threshold. We believe this demonstrates that our quantum wire laser is significantly more stable in emission energy versus pump power than are corresponding 2D quantum well lasers.

At this time, the weaknesses of these 1D lasers are that:

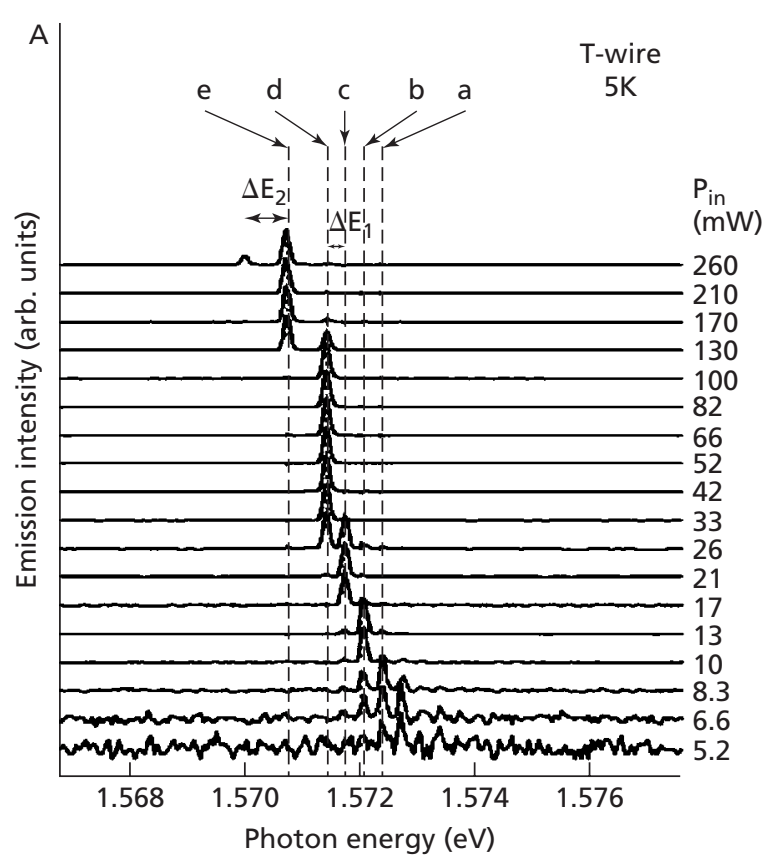

The normalized output spectra are shown for input powers ranging from the $5.2 \mathrm{~mW}$ threshold to $260 \mathrm{~mW}$.

Figure 13.

Lasing spectra of the single $T$-wire at $T=5 K$.

- They are complicated to fabricate in large numbers, and

- They operate only below about $100 \mathrm{~K}$, because of the small quantum wire confinement energy compared to the confinement in the nearby stem and cross-arm quantum wells.

There is ongoing research in our laboratory to address these issues.

\section{References}

[1] A. R. Goñi, L. N. Pfeiffer, K. W. West, A.

Pinczuk, H. U. Baranger, and H. L. Stormer, "Obsevation of Quantum Wire Formation at Intersecting Quantum Wells," Appl. Phys. Lett., 61 (1992), 1956-1958.

[2] Y. Hayamizu, M. Yoshita, S. Watanabe, H. Akiyama, L. N. Pfeiffer, and K. W. West, “Lasing from a Single-Quantum Wire," Appl. Phys. Lett., 81:26 (2002), 4937-4939.

[3] R. Held, T. Heinzel, A. P. Studerus, K. Ensslin, and M. Holland, "Semiconductor Quantum Point Contact Fabricated by Lithography with an Atomic Force Microscope," Appl. Phys. Lett., 71:18 (1997), 2689-2691. 
[4] D. Natelson, R. W. Willett, K. W. West, and L. N. Pfeiffer, "Fabrication of Extremely Narrow Metal Wires," Appl. Phys. Lett., 77:13 (2000) 1991-1993.

[5] D. Natelson, R. W. Willett, K. W. West, and L. N. Pfeiffer, "Geometry-Dependent Dephasing in Small Metallic Wires," Phys. Rev. Lett., 86:9 (2001), 1821-1824.

[6] L. P. Rokhinson, V. Larkina, Y. B. Lyanda-Geller, L. N. Pfeiffer, and K. W. West, "Spin Separation in Cyclotron Motion," Phys. Rev. Lett., 93:14 (2004), 146601-1-146601-4.

[7] L. P. Rokhinson, D. C. Tsui, L. N. Pfeiffer, and K. W. West, "AFM Local Oxidation Nanopatterning of a High Mobility Shallow 2D Hole Gas," Superlattices Microst., 32:2 (2002), 99-102.

[8] W. Wegscheider, L. N. Pfeiffer, M. M. Dignam, A. Pinczuk, K. W. West, S. L. McCall, and R. Hull, "Lasing from Excitations in Quantum Wires," Phys. Rev. Lett., 71:24 (1993), 4071-4074.

\section{(Manuscript approved June 2005)}

LOREN N. PFEIFFER is a distinguished member of

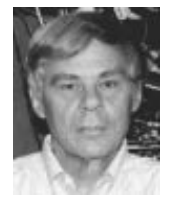
technical staff in the Semiconductor Physics Research Department at Bell Labs in Murray Hill, New Jersey. He received his B.S. degree in physics from the University of Michigan in Ann Arbor and his Ph.D., also in physics, from The Johns Hopkins University in Baltimore, Maryland. A world-renowned leader in the field of molecular beam epitaxy (MBE) technology, he is currently focusing on high-purity growth of GaAs/AIGaAs quantum well heterostructures. His devices have been proven to exhibit the highest electron mobility ever reported for any semiconductor. His professional interests include ultra-clean semiconductor growth using $M B E$, as well as studies of quantum wires, quantum dots, and other nanoscale structures with novel properties. Dr. Pfeiffer is a fellow of both the American Physical Society and the Johns Hopkins Society of Scholars, and he is the recipient of the 2004 McGroddy Prize of the American Physical Society.

KENNETH W. WEST is a member of technical staff in

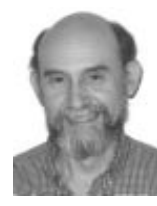
the Semiconductor Physics Research Department at Bell Labs in Murray Hill, New Jersey. He is currently working on the growth and characterization of novel semiconductor structures. His professional interests include development of new molecular beam epitaxy processes for high-purity semiconductor structures. He holds a B.S. degree in physics from Bucknell University in Lewisburg, Pennsylvania.

ROBERT L. WILLETT is a member of technical staff in

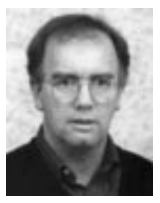
the Semiconductor and Physics Research Department at Bell Labs in Murray Hill, New Jersey, where he conducts research on condensed matter systems. He received an S.B degree in physics from the Massachusetts Institute of Technology (MIT) in Cambridge, and M.D. from the University of California at San Francisco, and a Ph.D., also in physics, from MIT. A fellow of the American Physical Society, Dr. Willett was a co-recipient of the 2002 Oliver Buckley Prize. His professional interests are in the physics of lower dimensional electron systems and nanostructures.

HIDEFUMI AKIYAMA is a visiting scientist (consultant)

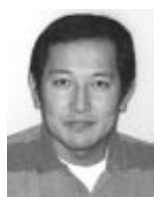
in the Semiconductor Physics Research Department at Bell Labs in Murray Hill, New Jersey, where he works on development, characterization, and optical investigation of T-shaped quantum-wire lasers. He received B.S. and Ph.D. degrees in physics from the University of Tokyo in Japan. Dr. Akiyama has been an associate professor in the Institute for Solid State Physics (ISSP) at the University of Tokyo since 1996.

LEONID P. ROKHINSON is a professor in the Department

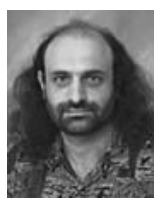
of Physics at Purdue University in West Lafayette, Indiana. He holds a Ph.D. in physics from the State University of New York at Stony Brook. Dr. Rokhinson, who is professionally interested in the physics of low dimensional systems, was the recipient of the 2004 National Science Foundation Career Award. 